\title{
lonizing Radiation Effects Modelling in Cells Population with Gold Nanoparticles
}

\author{
Hamizah Rashid ${ }^{a}$, Fuaada Mohd Siam ${ }^{\mathrm{a}, *}$, Normah Maan ${ }^{\mathrm{a}}$, Wan Nordiana W Abd \\ Rahman $^{\text {b }}$ \\ a Department of Mathematical Sciences, Faculty of Science Universiti Teknologi \\ Malaysia, 81310 Johor Bahru, Malaysia; ${ }^{b}$ School of Health Sciences, Universiti Sains \\ Malaysia, Kubang Kerian, Kelantan, 16150 Kota Bharu, Malaysia
}

\begin{abstract}
Radiosensitizer such as gold nanoparticle is a promising agent to be used in radiotherapy to increase the number of cancer cell death. Gold nanoparticle increases the production of the secondary electron after being hit by primary radiation that will cause DNA damage. The gold nanoparticle can be targeted to specific cancer cells and therefore reduce damage to the healthy nearby cell. Thus, nanoparticles will elevate the efficacy of radiation treatment without delivering a high radiation dose that will damage the organ at risk. Therefore, this paper aimed to study the effects of radiosensitizer on radiation therapy. The study was done by incorporating the function of dose deposited by gold nanoparticles into the existing model of ionizing radiation effects. The model was mathematically described using Ordinary Differential Equations (ODEs). The simulation results were fitted to the Linear Quadratic (LQ) formulation to give the ratio for $\alpha / \beta$. Next, the parameter estimation and sensitivity analysis of the model are carried out using experimental data of HeLa cell with the aid of the MATLAB programming. The estimated parameter values can explain the radiobiology process, which can support the result of the experimental design. The result showed that the sum-squared error (SSE) between simulation data and experimental data obtained is 0.015 which indicates an excellent fit to the experimental data. Thus, this model is in line with the experimental result. The model is able to explain the dynamics process of ionizing radiation effects with gold nanoparticles on the cell population.
\end{abstract}

Keywords: lonizing Radiation, Nanoparticle, Double-Strand Breaks (DSBs), Parameter Estimation, Sensitivity Analysis

*For correspondence: fuaada@utm.my

Received: 30 Nov 2020

Accepted: 11 October 2021

(C) Copyright Rashid. This article is distributed under the terms of the Creative Commons Attribution License, which permits unrestricted use and redistribution provided that the original author and source are credited

\section{Introduction}

A cancer cell is a cell that divides out of control. Therefore, to stop the spreading of cancer cells, ionizing radiation is applied. Radiotherapy used high-energy radiation such as photons and electron beams to destroy cancer cells. The beams are produced by a linear accelerator (LINAC) to treat various types of malignant diseases. Nevertheless, radiotherapy is always limited by the existence of surrounding healthy tissues that constraint the lethal delivery to the targeted cell.

Therefore, an approach has been made to stimulate cell sensitivity in radiation oncology through the introduction of radiosensitizer. Radiosensitizer is a metal used to increase the ionizing radiation effect. Metal with a high atomic number (Z), especially nanoparticles, absorb high radiation and allowing for better targeting the cell. These combinations limit the damage to normal cells and enhance radiation treatment. For the past few years, nanoparticles such as gold and silver became one of the most investigated and explored $[1,2,3]$. 
Recently, Yang et al. [4] implemented gold nanoparticles to enhance radiation dose in cancer treatment to overcome the side effects of chemotherapy while treating cancer. The result showed that the cancer cells' death is increased after receiving radiation dose of 2 Gy. Then, Khan et al. [5] tested different concentrations of nanoparticles on human breast cancer cells (MCF-7). Their result showed that nanoparticles can induce cell death without affecting the nearby cells. Therefore, these findings show that nanoparticles can be used to improve the efficacy of radiation treatment.

Other than the experimental work, a quantitative model is also beneficial to better understand the effect of nanoparticles and as a preliminary study before proceeds for treatment. To date, Cardilin et al. [6] proposed a mechanistic model to examine the effect of radiation and nanoparticles in tumour growth. The model consists of proliferating cells and cell damage following a combination of ionizing radiation and radiosensitizer. However, this model does not consider the properties of radiosensitizer, such as the size and density of radiosensitizer. A review done by Sah et al. [7] concluded that different types of molecular targeting require different size radiosensitizers. However, the physical interaction between radiation and nanoparticle is not well described.

In 2018, Siam et al. [8] designed a structured population model for double-strand breaks (DSBs) and misrepair cells following ionizing radiation. This model explained the cells dynamics process of the cell population following ionizing radiation which are death rate, repair rate and the probability of correctly cell's repair. However, this model only considered primary electrons of ionizing radiation as a source of DSBs formation which caused DNA damage.

Therefore, this study aims at developing a mathematical relation which represents the physical interaction between ionizing radiation and radiosensitizer and dose deposited by radiosensitizer. For this purpose, we modify an existing radiation effect model by Siam et al. [8]. At the end of the study, the number of cells survived following these combination agents is obtained, followed by performing parameter estimation and sensitivity analysis is carried out to identify which parameter influences the model output.

\section{Gold Nanoparticles in Radiation Research: Theory of photoelectric effect}

Nanoparticles have been proposed as a novel radiosensitizer agent due to their ability to induce toxicity to targeted cells and reduce effects to normal cells. This phenomenon can be explained through the formation of photoelectric. Photoelectric is the emission of electrons from the metal's surface in response to incident light. An electric current causes the ejection of the electron from its shell called secondary electron [9].

\section{Dose Deposited by Gold nanoparticle}

Among all nanoparticles, gold nanoparticles are promising as good enhancement agents in radiotherapy. According to Goa and Zheng [9], gold nanoparticles contain high electron volume density, thus generating more secondary electrons. Besides that, the gold nanoparticle has several properties. According to Huang et al. [10], nanoparticles are biocompatible which appears in different types and size, which can enhance the radiation effect over the large area. Also, low systemic clearance which allows the material to absorb into a targeted cell which can be specifically delivered to the tumour tissue. Besides that, the image of the gold nanoparticle is easy to quantify.

Several experiments have been conducted to investigate the influence of size nanoparticle on radiation effect. Hillyer and Albrecht [11] studied the cellular uptake with 4,10, 28 and $58 \mathrm{~nm}$ of metallic colloidal gold nanoparticle. They concluded that nanoparticle with $4 \mathrm{~nm}$ diameter showed higher accumulation in various tissue lines. The result obtained is in agreement with a few works which demonstrated that lower size of nanoparticle has a higher accumulation in cell tissue [10,11].

An article by Saion et al. [12] explained why the dose deposited by the small nanoparticle size shows 
the domination in radiation effect is due to the high number of nucleation processes over ion during the nanoparticle formation by gamma reduction. Nucleation is a process whereby reactive species such as hydrated electrons $\left(\mathrm{e}_{\mathrm{aq}}{ }^{-}\right)$reduced the nanoparticle ions into a zero-valent atom. For example, the reaction between the hydrated electron $\left(\mathrm{e}_{\mathrm{aq}}{ }^{-}\right)$and silver nitrate, $\left(\mathrm{Ag}^{+}\right)$, results in $\mathrm{Ag}^{0}$. The relationship between the average size of the particle with silver nitrate, $\left(\mathrm{Ag}^{+}\right)$, with a concentration of $4.2 \times 10^{-4} \mathrm{M}$, provided by Saion et al. [12] is shown in Figure 1. The figure shows that the particle size decreased with an increasing dose. Before, Leung et al. [13] studied the relationship between secondary electrons generated by goldnanoparticles (GNPs) with various size. The work is done by utilizing the Monte Carlo toolkit, which provides simulation of interaction between particles and matters. Their simulation results are in agreement with the article by Saion et al. [12].

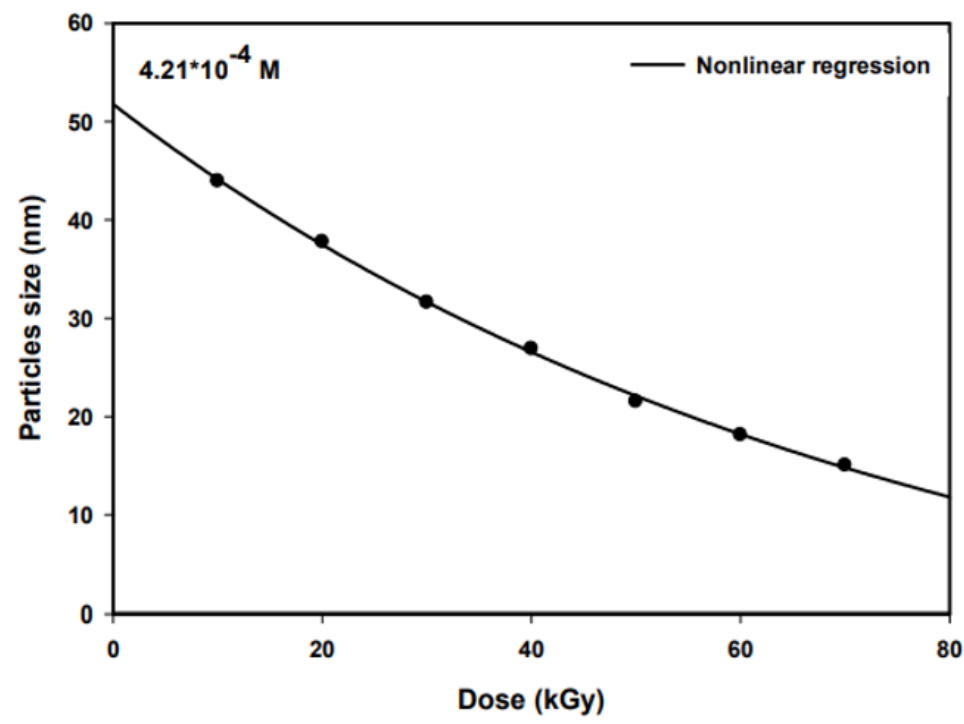

Figure 1. Average particle size versus dose for silver nitrate with a concentration of $4.2 \times 10^{-4} \mathrm{M}$ (Saion et al. [12]).

\section{Modelling Radiation Responses}

A few mathematical models have been derived to study the effect of ionizing radiation $[14,15,16]$. One of them is a mathematical model by Siam et al. [8]. Their work described the population dynamic of cells following ionizing radiation. The conventional radiotherapy gives effects to the targeted cell as well as nearby cell. Therefore, the efficacy of conventional radiotherapy can be improved by using a new methodology in order to maintain the surrounding cells from affected.

Nanoparticles have emerged as a suitable agent to deliver radiation doses specifically to the targeted cells without harming nearby cells. The properties of nanoparticles are taken into consideration to investigate the radiation efficacy. Many researches have been made to study nanoparticles' effect in cancer treatment, especially using Monte Carlo simulation $[17,18]$.

Meanwhile, studies by Saion et al. [12] and Leung et al. [13] showed that secondary electrons deposited by nanoparticles decreased as the size of nanoparticles increased. At the same time, the properties of nanoparticles, such as surface area and surface texture are considered in Simko et al. [18]. They obtained the same result as Saion et al. [12] and Leung et al. [13]. According to Štefančíková et al. [19], DSBs induced by nanoparticles are two times higher than DSBs induced by primary dose. Therefore, in this study, we will modify a mathematical model developed by Siam et al. [8] to study the effects of combination of ionizing radiation and nanoparticles. 
The modified model will consider the interaction between primary and secondary electrons by nanoparticles, which will induce double-strand breaks (DSBs) to cause lethal damage to the targeted cells. Following the simulation and experimental results in $[12,14,18]$, we assume that the dose deposited by gold nanoparticles decreased exponentially with the size of gold nanoparticles. An experiment done by Rashid et al. [20] used sphere gold nanoparticle to study the effect of ionizing radiation. Therefore, in this work, we assumed that the nanoparticle is sphere in order to describe the interaction between nanoparticle and ionizing radiation. In the next section we will use experimental data by Rashid et al. [20] to estimate the model parameter. Recently, Mori and Hengmann [25], implemented equation of volume of sphere to calculate the number of spherical gold nanoparticle in order to study the influence of nanoparticle's shape in radiation effect. Therefore, in this study, the amount of dose deposited by sphere gold nanoparticles can be fitted to an empirical relation of the form

$$
\text { Dose }_{\text {nano }}=\exp \left(-\frac{\text { radius of nanoparticle }}{\text { volume of gold nanoparticle }}\right),
$$

where the formula for the volume of sphere shape is given by, $\frac{4}{3} \pi r^{3}$, where $\pi$ is a constant value, 3.142 , and $r$ is the radius of gold nanoparticles. The above equation uses the spherical volume as the main subject in order to describe the interaction between radiation and nanoparticles. In general, the radiation dose is estimated of the deposited energy per mass of living matter, hence, we assume that the dose deposited by nanoparticle relies on the same concept to deposit the secondary electron. Therefore, equation (1) becomes

$$
\text { Dose }_{\text {nano }}=\exp \left(-\frac{\text { radius of nanoparticle }}{\text { volume of gold nanoparticle }}\right) * \text { Dose }_{\text {primary }} \text {. }
$$

Since the use of gold nanoparticles will increase the number of DNA damage, specifically the formation of DSBs, therefore the probability of DSB produced as in Siam et al. [8] need to be modified. Here, in this work we assume that the probability of DSBs produced follows a Poisson distribution with mean

$$
\lambda=\delta\left(\text { Dose }_{\text {primary }}+\text { Dose }_{\text {nano }}\right),
$$

where $\delta$ measure the radiosensitivity of the cell. The probability of a particular cell acquiring DSBs is given by

$$
P(\text { noDSBs }=k)=\frac{\lambda^{k} e^{-\lambda}}{k !}
$$

The cells population following ionizing radiation is governed by the following ordinary differential equation

$$
\begin{aligned}
\frac{d N_{k, m}}{d t} & =-\chi(k, m) N_{k, m}-\gamma(k) N_{k, m}+p(k+1) \gamma(k+1) N_{k, m}+ \\
& =(1-p(k+1)) \gamma(k+1) N_{k+1, m-1}
\end{aligned}
$$

This equation describes the repair rate, $\gamma(k)=\frac{\mathrm{V}_{\max } k}{K_{M}+k}$, and death rate, $\chi(k, m)=\alpha_{1} m+\alpha_{2} k^{2}$, for DNA damages induced by ionizing radiation. Repairing DSBs is not $100 \%$ success; therefore, the probability of correctly repaired is defined as, $p(k+1) \gamma(k+1) N_{k, m}$, and incorrectly repaired of chromosome rejoin is represented by the following term $(1-p(k+1)) \gamma(k+1) N_{k+1, m-1}$. The solution of the ODEs system at time, $t$, is given as 


$$
\mathrm{N}(t)=\mathrm{N}(0) e^{A t}
$$

where $N(0)$ is the initial population and $A$ is matrix defined by ODEs in Equation (5).

\section{Simulation Results}

By taking into consideration on secondary electrons deposited by gold nanoparticles implementing in Siam et al. [5], the simulation of the results is shown in Figure 2.

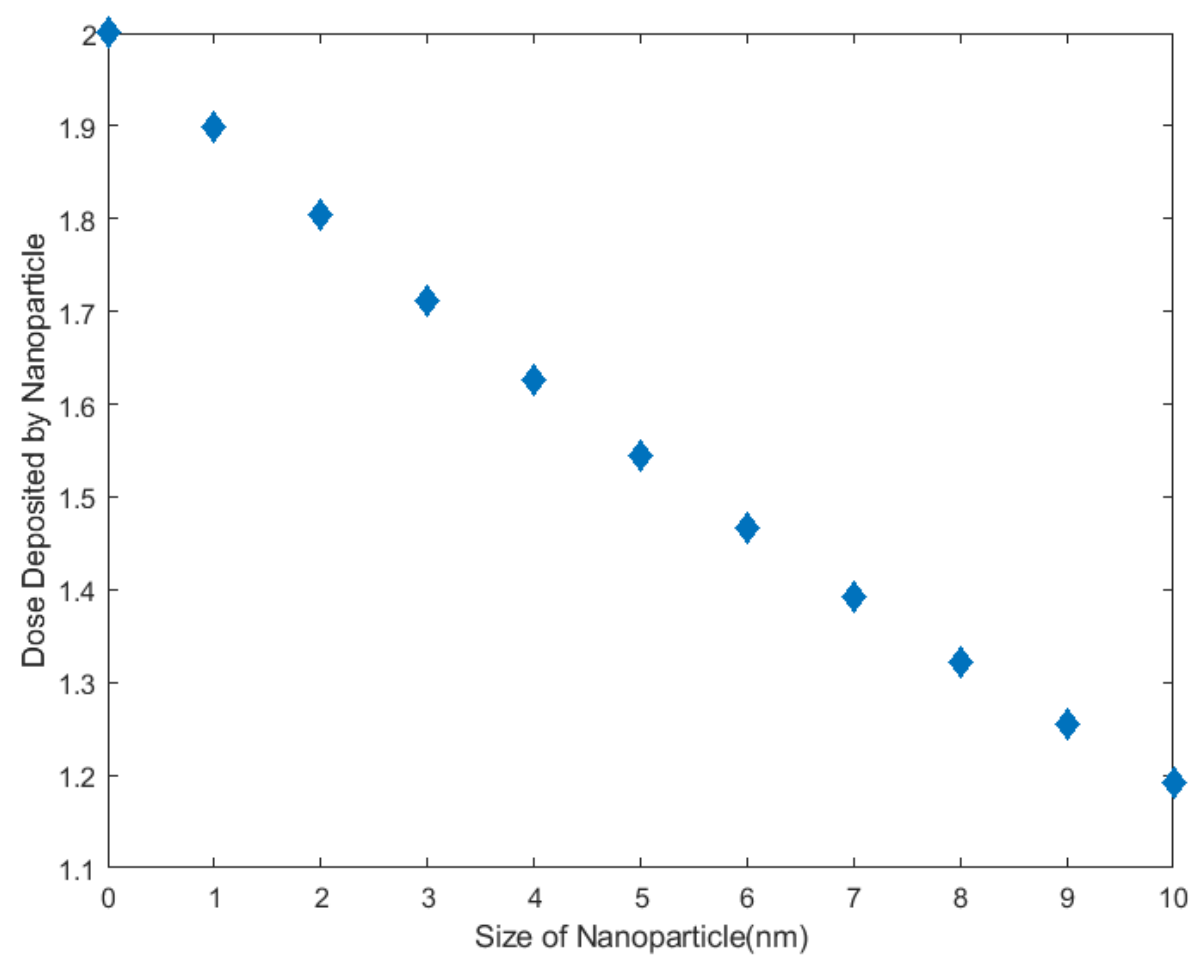

Figure 2. Dose deposited by different size of radius gold nanoparticle follows equation (2) with dose 2 Gy, and density of gold nanoparticle $19.32 \mathrm{~g} / \mathrm{cm}^{3}$.

Figure 2 shows the dose deposited by nanoparticles for the size of nanoparticles from $1 \mathrm{~nm}$ to $10 \mathrm{~nm}$. The size of nanoparticles is chosen for simulation purposes only. It can be seen that the dose deposited by gold nanoparticle is decreasing if the uptake size of nanoparticle increase. Therefore, our simulation result is in line with the experimental and simulation works which were obtained by the previous studies $[9,10,15]$. For radiation effects by primary electron and secondary electron from gold nanoparticles, we generate ten simulation data points for the value of the models' parameter as in Siam et al. [8], where, $\delta=2 G y^{-1}, \alpha_{1}=1.5 \mathrm{~h}^{-1}, \mathrm{p}=0.95, \alpha_{2}=0.01 \mathrm{~h}^{-1}, V_{\max }=1 \mathrm{~h}^{-1}$ and $K_{\max }=3 \mathrm{mM}$. These values and the total number of data are chosen as an example only. The result of the simulation is shown in Figure 3.

Figure 3 shows two survival fractions of the cell with gold nanoparticle and without gold nanoparticle following ionizing radiation. For combination dose with gold nanoparticles, we set the radius of gold nanoparticles, $r=5 \mathrm{~nm}$. It can be seen that from the simulation (asterisks) the survival fraction with gold nanoparticles is low, indicating a high number of cell death compared to the simulation without gold nanoparticles. The simulation data is then fitted to the LQ relation (solid line). The results explain the use of gold nanoparticle in radiation oncology increase number of cell death. Further analysis is carried out by performing parameter estimation using experimental data from Rashid et al. [20]. 


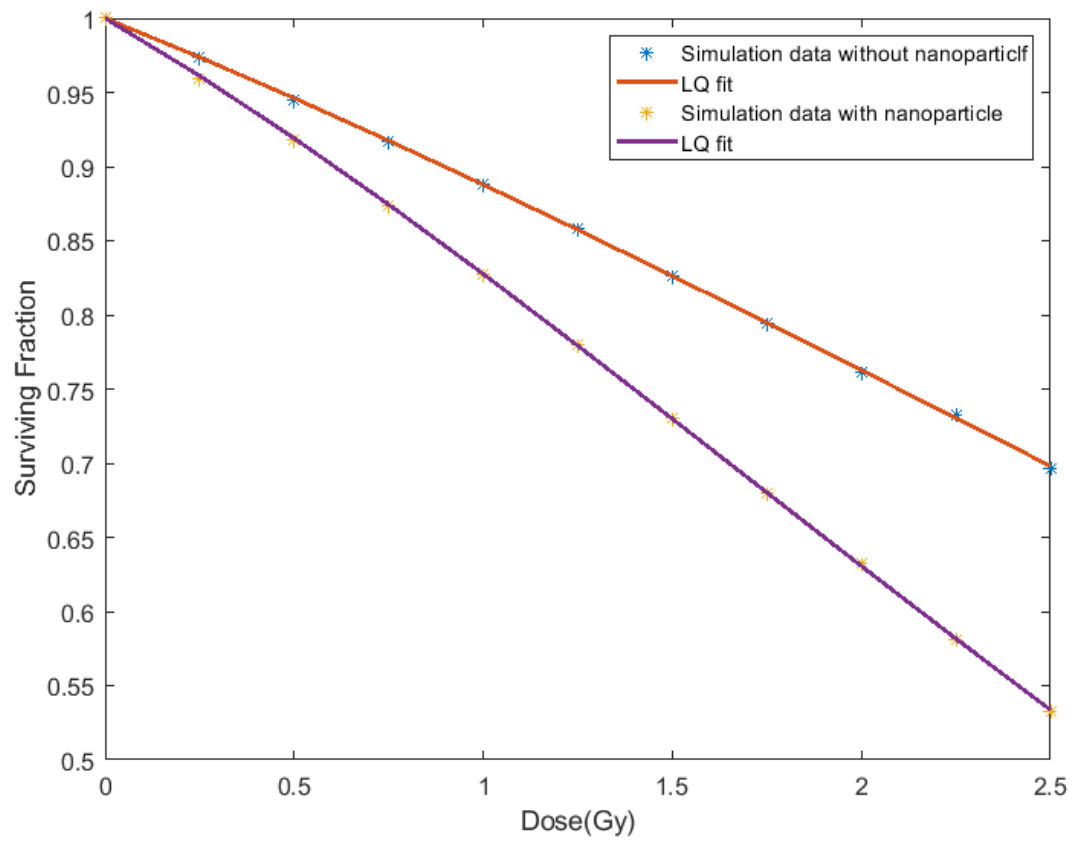

Figure 3. Simulation result for dose with gold nanoparticle and without gold nanoparticle.

\section{Parameter Estimation}

The model formulation in this paper contains six parameters: $\delta, \alpha_{1}, \alpha_{2}, p, V_{\max }, K_{\max }$. To estimate all the parameters value, we will incorporate experimental data of HeLa cells by Rashid et al. [17] into the model. The experiment is about the study of nanoparticles effect on HeLa cells with the concentration of gold nanoparticles, AuNPs, is $1 \mathrm{mMol} / \mathrm{L}$. Parameter estimation is carried out using Genetic Algorithm. This method generates solutions to optimize problems using techniques inspired by natural evolution, such as mutation, selection and crossover. In this study, we use Genetic Algorithm to estimate parameter value because it is efficient method that generates optimal solution over numerous possible solutions [21]. Figure 4 shows the flowchart of parameter estimation process, while Figure 5 shows the algorithm for Genetic Algorithm in performing parameter estimation.

In this study we set 20 iterations as a stopping criteria. The parameter estimation result is shown in Table 1. From the simulation, $\alpha / \beta$, the ratio value obtained is close to the experimental data which is 8.5 , where $\alpha$ and $\beta$ value are 0.16 and 0.02 , respectively.

The estimated values obtained are close to the original value used for simulation except for $\alpha_{1}$, which correspond to death rate of misrepair cells. Earlier, the value for $\alpha_{1}$ is set 1.5 for simulation purposes. In this case, the death rate for misrepair cells contributes more to cell death than the death rate associated with DSBs.

Table 2 presents the $95 \%$ confidence interval for the six parameters of the model. These confidence intervals are computed based on the estimated parameters obtained using ten bootstrap data sets. In this study, we believe that $95 \%$ of the model's parameters will fall in the proposed range. We use the estimated parameters in Table 1 to observe how the simulation fits the experimental data. See Figure 5.

Figure 5 shows the survival fraction for model simulation using estimated parameter value, experimental data and $L Q$ formulation. The surviving fraction between experimental data and model simulation slightly differs with sum-squared error (SSE) obtained is 0.015 , which corresponds to an excellent fit. 


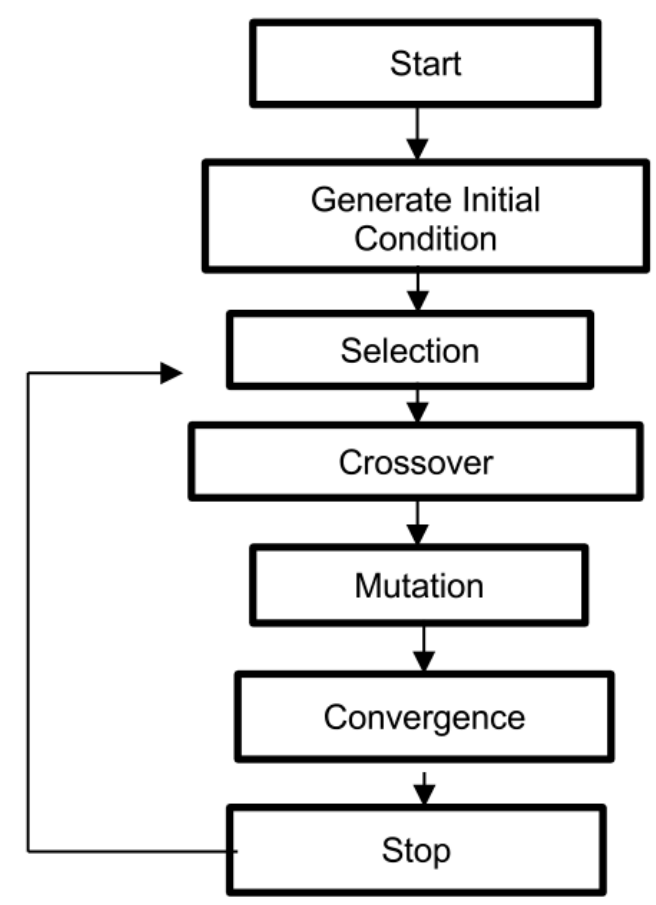

Figure 4. Flow chart of parameter estimation algorithm.

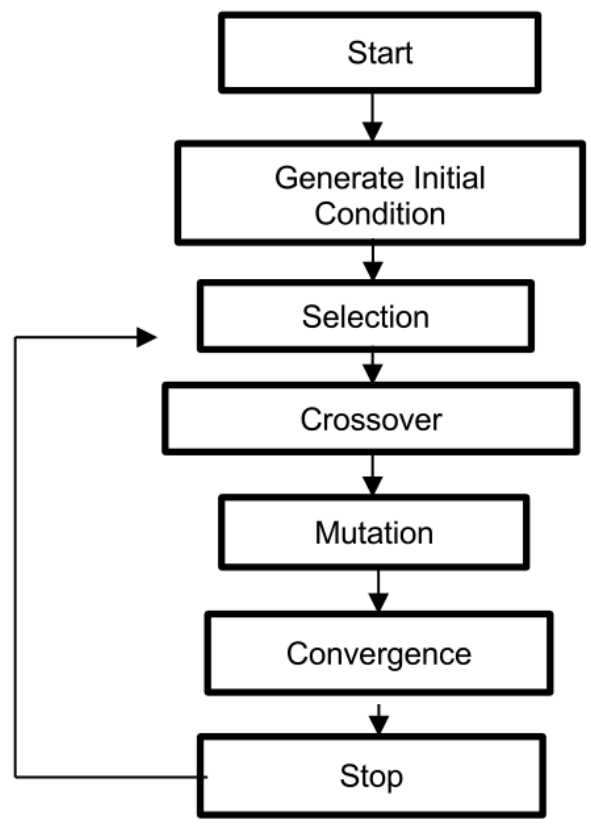

Figure 5. Flow chart of Genetic Algorithm. 
Table 1. Mean of the result parameter estimation value.

\begin{tabular}{cc}
\hline Parameter & Value(mean) \\
\hline$\delta$ & 2.15983 \\
$\alpha_{1}$ & 0.87165 \\
$\alpha_{2}$ & 0.00345 \\
$p$ & 0.91657 \\
$V_{\max }$ & 2.80903 \\
$K_{m}$ & 3.48357 \\
$\alpha$ & 0.16 \\
$\beta$ & 0.02 \\
Ratio of $\alpha / \beta$ & 8.0 \\
\hline
\end{tabular}

Table 2: The bootstrap 95\% confidence interval for the estimated parameters' value.

\begin{tabular}{cccc}
\hline Parameter & Value & $\begin{array}{c}\text { Standard } \\
\text { Error }\end{array}$ & $95 \%$ Bootstrap Cl \\
\hline$\delta$ & 2.15983 & 0.3102 & $(2.6048,3.2251)$ \\
$\alpha_{1}$ & 0.87165 & 3.7457 & $(9.1155,16.6069)$ \\
$\alpha_{2}$ & 0.00345 & 0.0008 & $(0.0002,0.0018)$ \\
$p$ & 0.91657 & 0.0129 & $(0.8909,0.9168)$ \\
$V_{\max }$ & 2.80903 & 0.5451 & $(1.2288,2.3189)$ \\
$K_{m}$ & 3.48357 & 0.5535 & $(3.1284,4.2354)$ \\
\hline
\end{tabular}

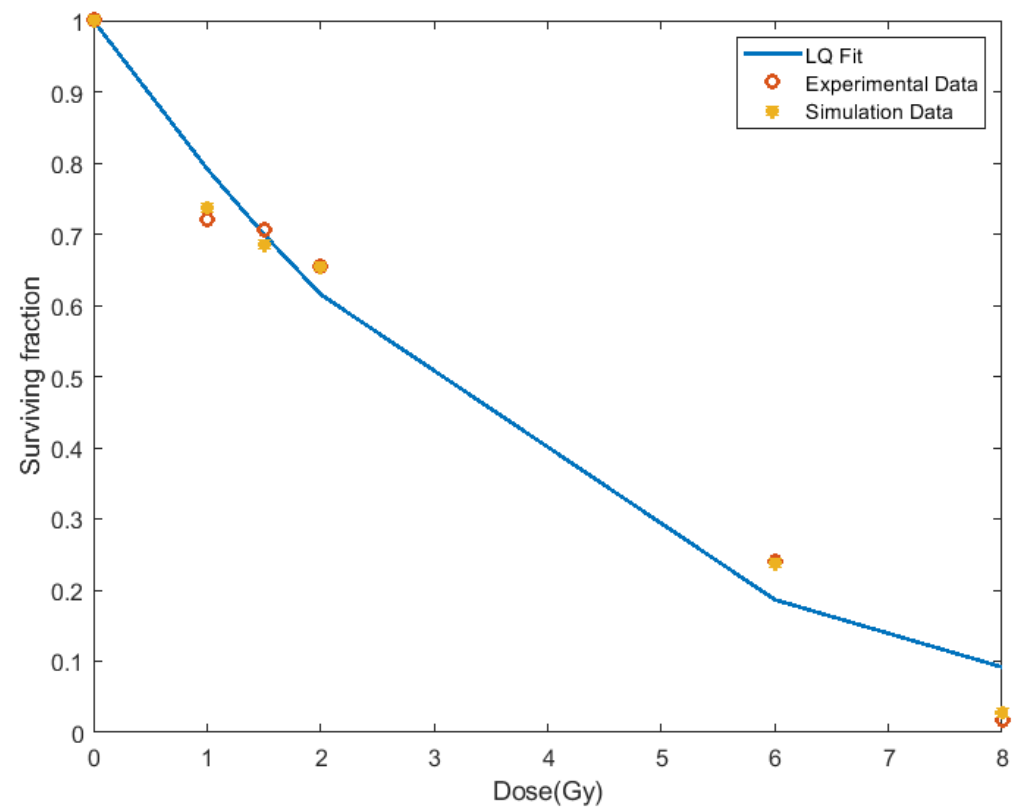

Figure 5: Simulation of HeLa cells using estimated parameter values estimated. 


\section{Sensitivity Analysis}

This section aims to analyze which parameter is sensitive to some modification in the parameter's value. We perform local sensitivity methods for this purpose. Local sensitivity analysis is a one at a time (OAT) method that analyses the model output by varying each model input parameter at one time while other input remains constant [22]. According to Nasir and Siam [23], the partial effect $S_{i}$ for each parameter, $p_{i}$, is calculated as a percentage:

$$
S_{i}=\frac{M\left(p_{i}, \ldots, p_{i} \times\left(1+f_{i}\right), \ldots, p_{e}\right)-M\left(p_{i}, \ldots, p_{i}, \ldots, p_{e}\right)}{f_{i}} \times \frac{p_{i}}{M\left(p_{i}, \ldots, p_{i}, \ldots p_{e}\right)},
$$

where $M\left(p_{i, \ldots} p_{e}\right)$ refers to the model function, $f_{i}$, is the fraction by which the parameter $p_{i}$ is changed. The parameter is increased by the fraction, $f_{i}$. At a time, we use the estimated parameter's value run by Genetic Algorithm. The partial effects are averaged to get the final products for each parameter. The result of sensitivity analysis is shown in Figure 6.

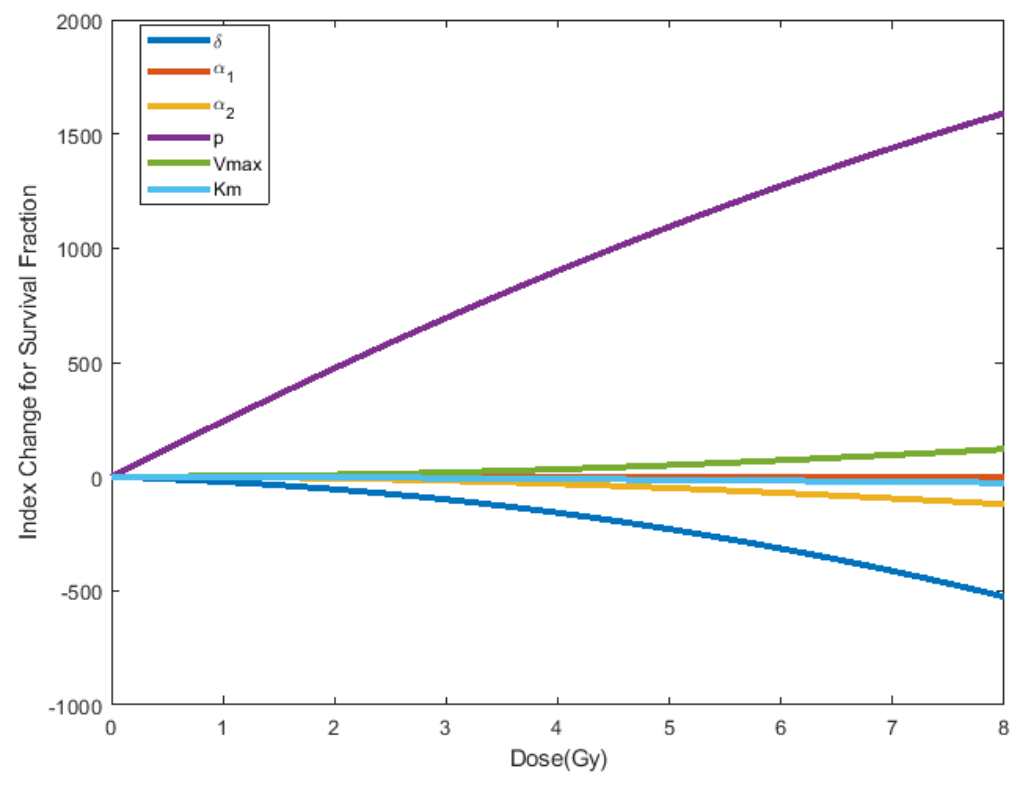

Figure 6: Index change for the model's parameter with perturbation of $1 \%$.

Figure 6 shows the result of the sensitivity analysis of the model's parameter. It is clearly seen that the probability of cell correctly repaired, $p$ is the most sensitive among other parameters. In this case, by increasing $1 \%$ value of, $p$, the survival fraction will increase and the ratio of $\alpha / \beta$ decreased from 8.5 to 8.4. In radiation oncology, the ratio represents the sensitivity of cell towards radiation, Van et al. [24]. Therefore, to ensure that more cells die after ionizing radiation, nanoparticles with a smaller radius can be used to increase cell sensitivity. As a result, the ratio of $\alpha / \beta$ increased correspond to high number of cell death of targeted cells.

\section{Conclusion}

The mechanistic model illustrates the factors that increase the amount of DNA damage produced. In this work, we consider the new technology implemented in curing cell cancer by introducing a radiosensitizer agent. The model suggested in this study addressed the combined effects of nanoparticles and ionizing 
radiation on the cell's population. The characteristics of radiosensitizer, such as size and radiosensitizer concentration, are considered in this work. The generation of secondary electrons by this agent shows an increase in the number of cell death. The results reflected the finding by Rashid et al. [17], which experimentally proved that gold nanoparticle induces radio sensitization effects. Model validation is done by performing estimating parameters using Genetic Algorithm. Parameter estimation is a procedure that searches the value of the model's parameter to match experimental data, which is done by minimizing the sum-squared error between model simulation data and experimental data. Estimated parameter values are then used to determine parameter sensitivities. This analysis is carried out to observe which parameters affect the survival fraction most. Overall, our results demonstrate that a strong effect on cell survival is the probability of cell correctly repaired, $p$. This model also allows quantitative characterizations of nanoparticles as a radiosensitizer to enhance the radiation effects. The same approach can be applied to evaluate other radiosensitizer agents functioning after exposure to radiation, including water molecules.

\section{References}

[1] S. Penninckx, A.C. Heuskin, C. Michiels, S. Lucas, Gold nanoparticles as a potent radiosensitizer: a transdisciplinary approach from physics to patient. Cancers, vol. 12, no. 8, pp. 2021.2020.

[2] S. H. Lim, C. H. Li, Y. I. Jeong et al., Enhancing radiotherapeutic effect with nanoparticle-mediated radiosensitizer delivery guided by focused gamma rays in lewis lung carcinoma-bearing mouse brain tumor models. International Journal of Nanomedicine, vol. 14, pp. 8861, 2019.

[3] S. Yang, G. Han, Q. Chen et al., Au-Pt Nanoparticle Formulation as a Radiosensitizer for Radiotherapy with Dual Effects. International Journal of Nanomedicine, vol. 16, pp. 239, 2021.

[4] C. Yang, K. Bromma, Di Ciano-Oliveira et al., Gold nanoparticle-mediated combined cancer therapy, Cancer Nanotechnology, vol. 9, no. 1, pp. 4,2018.

[5] F. Khan, S. Akhtar, S. Almofty et al., MSP-nanoparticles induced cell death on human breast adenocarcinoma cell line (MCF-7 Cells): morphometric analysis, Biomolecules, vol. 8, no. 2, pp. 32, 2018.

[6] T. Cardilin, J. Almquist, M. Jirstrand et al., Model-Based Evaluation of Radiation and Radiosensitizing Agents in Oncology, CPT: Pharmacometrics \& Systems Pharmacology, vol. 7, no. 1, pp. 51-58.2018.

[7] B. Sah and MP. Antosh, Effect of size on gold nanoparticles in radiation therapy: Uptake and survival effects. Journal of Nanomedicine, vol. 2, no. 1, pp. 1013, 2019.

[8] S.M. Siam, M. Grinfeld, A. Bahar et al., A mechanistic model of high dose irradiation damage, Mathematics and Computers in Simulation, vol.151, pp. 156- 168, 2016.

[9] J. Gao and Y. Zheng, Monte Carlo study of secondary electron production from gold nanoparticle in proton beam irradiation. International Journal of Cancer Therapy and Oncology, vol 2, pp. 2025, 2014.

[10] K. Huang, H. Ma, J. Liu et al., Size-Dependent Localization and Penetration of Ultrasmall Gold Nanoparticles in Cancer Cells, Multicellular Spheroids, and Tumors in Vivo. ACS Nano, vol. 6, no. 5, pp. 4483-4493,2012.

[11] J. F. Hillyer and R. M. Albrecht, Gastrointestinal prescription and tissue distribution of differently sized colloidal gold nanoparticles. Journal of Pharmaceutical Sciences, vol. 90, no. 12, pp. 1927-1936, 2001.

[12] E. Saion, E. Gharibshahi, K. Naghavi, Size-controlled and optical properties of monodispersed silver nanoparticles synthesized by the radiolytic reduction method. International Journal of Molecular Sciences, vol. 14, no. 4, pp. 7880-7896, 2013.

[13] M. K. Leung, J. C. Chow, B. D. Chithrani et al, Irradiation of gold nanoparticles by x-rays: Monte Carlo simulation of dose enhancements and the spatial properties of the secondary electrons production, Medical Physics, vol. 38, no. 2, pp. 624-631, 2011.

[14] J. M. Wentz, V. Vainstein, D. Oldson et al., Mathematical model of radiation effects on thrombopoiesis in rhesus macaques and humans, Journal of Theoretical Biology, vol. 383, pp. 44-60, 2015.

[15] T. Wada, Y. Manabe and M. Bando, A mathematical model for the effects of radiation to the induced cancer in mice, In APS March Meeting Abstracts, pp. Y6-012, 2017.

[16] Y. Watanabe, E. L. Dahlman, K.Z. Leder \& S. K. Hui, A mathematical model of tumor growth and its response to single irradiation, Theoretical Biology and Medical Modelling, vol. 13, no. 1, pp.1-20, 2016.

[17] J. C. Chow, M. K. Leung, S. Fahey et al., Monte Carlo simulation on low-energy electrons from gold nanoparticle in radiotherapy, In Journal of physics: Conference series, vol. 341, no. 1, pp. 012012. IOP Publishing. 2012.

[18] M. Simkó, D. Nosske W. and Kreyling, Metrics, dose, and dose concept: the need for a proper dose concept in the risk assessment of nanoparticles. International Journal of Environmental Research and Public Health, vol. 11, no. 4, pp. 4026-4048, 2014.

[19] L. Štefančíková, S. Lacombe, D. Salado et al., Effect of gadolinium based nanoparticles on nuclear DNA damage and repair in glioblastoma tumor cells. Journal of nanobiotechnology, vol. 14, no. 1, pp. 1-15, 2016.

[20] R. A. Rashid, K. A. Razak, M. Geso et al., Radiobiological Characterization of the Radiosensitization Effects 
by Gold Nanoparticles for Megavoltage Clinical Radiotherapy Beams. BioNanoScience, vol. 8, no. 3, pp.713722, 2018.

[21] P. Bajpai and M. Kumar, Genetic algorithm-an approach to solve global optimization problems. Indian Journal of Computer Science and Engineering, vol. 1, no. 3, pp. 199-206,2010.

[22] J. Morio, Global and local sensitivity analysis methods for a physical system, European Journal of Physics, vol. 32, no. 6, pp. 1577,2011.

[23] M.H. Nasir and F. M. Siam, Simulation and Sensitivity Analysis on the Parameter of Non-Targeted Irradiation Effects Model, Jurnal Teknologi , vol. 81, no.1, pp. 133-142.2019.

[24] C. M. Van Leeuwen, A. L. Oei, J. Crezee et al., The alfa and beta of tumours: a review of parameters of the linear-quadratic model, derived from clinical radiotherapy studies, Radiation Oncology, vol. 13, no. 1, pp. 96, 2018.

[25] T. Mori and T. Hegmann, Determining the composition of gold nanoparticles: a compilation of shapes, sizes, and calculations using geometric considerations, Journal of Nanoparticle Research, vol. 18. no. 10, pp. 1-36, 2016. 